Recibido: 6 febrero 2018 | Aceptado: 25 octubre 2018 | Publicado: 20 diciembre 2018 Cita: Guerrero González, S. (2018). Los reguladores no verbales de inicio y de cierre en secuencias estructurales de narraciones conversacionales: un estudio sociopragmático. Normas, 8, 184-200. doi: https://doi.org/10.7203/Normas.v8i1.11494

\title{
LOS REGULADORES NO VERBALES DE INICIO Y DE CIERRE EN SECUENCIAS ESTRUCTURALES DE NARRACIONES CONVERSACIONALES: UN ESTUDIO SOCIOPRAGMÁTICO'
}

\author{
NONVERBAL REGULATORS IN STRUCTURAL SEQUENCES INITIATION AND CLOSURE \\ OF CONVERSATION NARRATIVES: A SOCIOPRAGMATIC STUDY
}

\section{Silvana Guerrero González}

Universidad de Chile

Resumen

En este estudio se indaga en el comportamiento de los reguladores no verbales de inicio y de cierre de secuencia estructural en narraciones conversacionales, con base en 54 narraciones conversacionales construidas por parejas de hablantes de Santiago de Chile, según las variables sexo y grupo socioeconómico. A partir de un análisis inductivo de los datos y siguiendo la propuesta inicial de Guerrero (2017b), se propone una categorización de reguladores no verbales de cierre de secuencia estructural narrativa, se intenta determinar su distribución de empleo en la cadena narrativa y se los relaciona con las variables sexo y grupo socioeconómico. Por último, se contrasta el empleo de reguladores no verbales de inicio y de cierre de secuencia estructural en la narración. El trabajo permite concluir que existen 38 reguladores de cierre claramente definidos. Se destaca especialmente el papel que tienen las miradas en todas las secciones de la narración y es relevante, además, que existan algunos reguladores no verbales muy frecuentes y diversos en el clímax del relato. Los hallazgos permiten verificar también que hay variación en el uso de este tipo de reguladores, pues, en general, son más frecuentes entre las parejas mujer-mujer y entre los hablantes de los grupos bajo y medio de la escala social. Por último, se comprueba que los reguladores de cierre son más diversos y más frecuentes que los de inicio de secuencia estructural en las narraciones, y que son más utilizados en la orientación y en la complicación de la narración.

Palabras clave: comunicación no verbal, reguladores, narraciones conversacionales, estructura narrativa, variación discursiva.
Abstract

This study investigates the behavior of nonverbal regulators of beginning and closure of structural sequence in conversational narratives, based on 54 conversational narratives constructed by couples of speakers of Santiago, Chile, in relation to the factors of sex and socioeconomic group. From an inductive analysis of the data and following Guerrero's initial proposal (Guerrero, 2017b), a categorization of nonverbal regulators of narrative structural sequence closure is proposed, an attempt is made to determine their distribution in the narrative sequence and they are associated to sex and socioeconomic group variables. Finally, the use of nonverbal regulators of the beginning and closing of structural sequence in the narrative is contrasted. The work allows to conclude that there are 38 of well-defined sequential regulators. The role of glances in all sections of the narrative is especially highlighted and it is also relevant that there are some very frequent and diverse nonverbal regulators at the climax of the story. The findings also verify that there is variation in the use of this type of regulators, since, in general, they are more frequent among female-female couples and among speakers of the low and middle socioeconomic groups. Finally, it is verified that the closing regulators are more diverse and more frequent than those of beginning of structural sequence in the narrations, and that they are more used in the orientation and in the complication of the narration.

KEY WORDS: nonverbal communication, regulators, conversation narratives, narrative structure, discourse variation

\footnotetext{
${ }^{1}$ Esta investigación se enmarca en el Proyecto FONDECYT 11150007, cuyo propósito es estudiar la co-construcción de narraciones de experiencia personal en relación con los factores génerolectal y sociolectal, en una muestra de hablantes de Santiago de Chile. El estudio se realizó en el contexto de una estancia investigación en el Departamento de Filología, Comunicación y Documentación de la Universidad de Alcalá, dentro del Programa "Giner de los Ríos", convocatoria curso 2017/18, bajo la tutoría de la Dra. Ana Cestero Mancera.
} 


\section{INTRODUCCIÓN}

En esta investigación se toman como base los postulados de Cestero (2004, 2009, 2014 y 2017), quien señala que la comunicación no verbal constituye una compleja área del lenguaje, en la cual están implicados todos los elementos culturales y los signos no verbales de los que disponen los hablantes en el proceso comunicativo. A la vez, se consideran los supuestos teórico-metodológicos esenciales de la sociolingüística variacionista e interaccional, con el propósito de indagar en el comportamiento de los reguladores no verbales de inicio y de cierre de secuencia estructural en narraciones conversacionales, sobre la base de 54 narraciones co-construidas (conversacionales o interaccionales) elaboradas por parejas de hablantes de Santiago de Chile, estratificadas según las variables sexo (hombre-hombre, hombre-mujer y mujer-mujer) y grupo socioeconómico (alto, medio y bajo). A partir de un análisis inductivo de los datos y siguiendo la propuesta inicial de Guerrero (2017b), donde se estudian los reguladores no verbales de inicio de secuencia estructural narrativa, se propone una categorización de reguladores no verbales de cierre de las mismas, en las mencionadas narraciones. Luego, se intenta determinar la distribución de dichos reguladores no verbales presentes en la cadena estructural de las narraciones y se los relaciona con las variables sexo y grupo socioeconómico. Por último, se contrasta el empleo de reguladores no verbales de inicio (Guerrero, 2017b) y de cierre de secuencia estructural en la narración. Para llevar a cabo este trabajo, se siguen, entre otros, los postulados de Juzwik (2012), quien pone énfasis en que las narrativas no deben elicitarse en entrevistas, sino que se debe atender al comportamiento interaccional en el que se despliegan.

Tal como se precisa en Guerrero (2017a, 2017b), la investigación propuesta se apoya en el interés que tienen las narraciones como configuradoras de la realidad social y cultural (Thornborrow y Coates, 2005), pero más concretamente en que, como sostienen Norrick (2000) y Sidnell (2010), por medio de la narración, los hablantes expresan información respecto de cómo conciben el mundo y la realidad observable. En este orden, la comunicación no verbal sería también una forma de expresión de los propios conceptos identitarios y culturales de los hablantes. Con esta investigación, además, se intenta seguir profundizando en el conocimiento del que se dispone sobre el proceso de construcción narrativa y la narración resultante, desde un ámbito que hay sido muy poco explorado, en general, y prácticamente no estudiado en el español de Chile, en particular: el de la comunicación no verbal desde una perspectiva sociopragmática.

\section{MARCO TEÓRICO}

\subsection{La narración de experiencia personal conversacional}

Como se ha señalado en múltiples investigaciones, Labov y Waletzky (1967) y Labov (1972) han demostrado ser por mucho el modelo de más alto rendimiento empírico en la investigación sobre la narrativa oral. Labov (1972: 360) define la narración como "one method of recapitulating past experience by matching a verbal sequence of clauses to the sequence of events which (it is inferred) actually occurred", y distingue entre relatos

\footnotetext{
2 Tanto el marco teórico como la metodología de esta investigación, reproducen, en buena parte, aunque con actualizaciones, los de Guerrero (2017b). 
mínimos, compuestos de -al menos- dos cláusulas narrativas en pasado y relatos completos, que exhiben seis rasgos estructurales claramente definidos:

1. Resumen: las narraciones se introducen, aunque no siempre, a través de un resumen que, generalmente, consiste en una proposición general que la narración va a ejemplificar.

2. Orientación: una narración prototípica comienza haciendo referencia a un tiempo, un lugar, unas personas y una conducta esperada en cierta situación.

3. Acción de complicación de la narración: corresponde al núcleo o esqueleto de la narración en el que aparecen los distintos eventos narrados, constituyendo el clímax del relato.

4. Evaluación: es utilizada por el narrador para validar su narración, es decir, para dejar en claro cuál es la razón de ser de su relato y su meta al narrarlo. La evaluación puede ser transversal al relato y no constituye estrictamente una parte, sino que está conformada por todos los fragmentos en que el narrador utiliza medios que hacen de la historia un relato interesante.

5. Resultado o resolución: la resolución dice lo que ocurrió finalmente, por lo tanto, está marcada por cláusulas narrativas; de hecho, en el desenlace aparece la última de ellas.

6. Coda: se trata de la moraleja. Generalmente, una cláusula narrativa lleva implícita una pregunta "y entonces, ¿qué ocurrió?". Cuando las preguntas que van surgiendo quedan respondidas, puede aparecer la coda, con la que el narrador regresa al presente, haciéndole saber a su oyente que la narración ha concluido (adaptado de Labov y Waletzky, 1967: 354-396 y Labov, 1972: 360)³.

Esta propuesta ha sido aplicada a múltiples investigaciones que abordan la construcción del relato oral que se obtiene a partir de la entrevista sociolingüística. No obstante, la narración conversacional ha sido muy poco investigada desde el enfoque variacionista. Por lo tanto, este estudio propone que es fundamental desarrollar trabajos con una metodología más cercana al estudio de la conversación cotidiana y que la narración coconstruida podría ser un tipo de relato similar al que aparece en el intercambio comunicativo diario. A este respecto, van Dijk (1997) precisa que las historias conversacionales son a menudo producidas en conjunto por varios narradores. Desde este punto de vista, la narración co-construida con la que se trabaja en esta investigación es interaccional, ya que se trata de una secuencia interactiva que se produce en el marco de la conversación coloquial. Dolón (1998), basándose en Cheepen (1988), caracteriza la historia conversacional con cuatro elementos clave: a) presencia de una secuencia coherente de estado $1>$ acontecimiento > estado 2, b) especificación de los participantes, c) ubicación temporal y d) componente evaluador.

Los trabajos que se centran en la narración interaccional en lingüística lo hacen desde el análisis del discurso y el análisis de la conversación. En este orden, Tusón (1995) concibe la conversación como una forma de discurso organizada a partir de la alternancia de turnos. Esta propuesta se aplica a la vez al relato conversacional, puesto que se trata de una

\footnotetext{
${ }^{3}$ Autores como Moreno Fernández (2009) se refieren al resumen como inserción. La coda y el resultado (o resolución) tienen funciones que coinciden parcialmente. La primera señala explícitamente que la historia ha terminado, a través de expresiones como "Y eso fue todo" (Silva-Corvalán, 2001). Por su parte, el resultado señala lo que aconteció finalmente. 
secuencia discursiva interactiva. Goodwin (2007), en tanto, destaca la importancia de estudiar la construcción interaccional de los relatos, pues los narradores van negociando el significado a medida que la historia se desarrolla. También Norrick (2000) señala que la co-construcción modula la relación personal de variadas formas, no solo porque los participantes reviven experiencias comunes, sino también porque los une y redunda en sentimientos de pertenencia a un grupo. Asimismo, Andrews et al. (2013) destacan la importancia de estudiar las narrativas desde múltiples enfoques. Uno de estos enfoques es, según los autores, el de la co-construcción, puesto que permite observer los códigos sociales y co-construidos, que reflejan expresiones de estados cognitivos o afectivos internos.

\subsection{La comunicación no verbal: los reguladores}

Los sistemas de comunicación son tres: lingüísticos, paralingüísticos y quinésicos. Los dos últimos constituyen la comunicación no verbal. Tomando como base los postulados de Cestero (2016a), en este trabajo se sostendrá que dentro del sistema paralingüístico se ubican las cualidades físicas del sonido (tono, timbre, intensidad u otras), las reacciones fisiológicas (risa, llanto, suspiros, entre otras), los elementos cuasi-léxicos (como las consonantizaciones) y la ausencia de sonido. En lo que dice relación con el sistema quinésico, se hallan gestos faciales y corporales, maneras (movimientos, toma de posiciones, etc.) y posturas (o posiciones estáticas). Existen, además, sistemas de comunicación no verbal culturales, a saber, la proxémica y la cronémica.

Como características de la comunicación no verbal, Cestero (2016a) incluye que: 1) comunica activa o pasivamente, 2) es funcional, 3) es plurifuncional, 4) es susceptible de variación, 5) se puede combinar con la comunicación verbal y 6) comunica y regula el mensaje. Consecuentemente con (4) y (6), en esta investigación se postula que existe una serie de reguladores que marca el cierre de cada parte de la cadena estructural narrativa (Labov y Waletzky, 1967; Labov, 1972; Labov, 2013), que son variables y susceptibles de clasificación y caracterización, pues el relato -tal como la comunicación en general- se regula y estructura mediante dichos mecanismos.

En esta línea, Ekman y Friesen (1969) clasifican en cinco tipos genéricos los actos no verbales: emblemas, ilustradores, reguladores, adaptadores y expresiones $\mathrm{O}$ manifestaciones de afecto o emoción. Alvarez (2003), por su parte, precisa que Ekman (1973) se referirá, dentro de los movimientos faciales, a los emblemas, ilustradores y reguladores como 'conversational signals' (o 'señales de conversación') para distinguirlos de las expresiones de emoción. Los reguladores no verbales sirven para organizar el flujo de la conversación, puesto que reclaman, entregan, retienen o ceden los turnos conversacionales. Además, le indican al interlocutor si debe continuar, apresurarse, repetir o prestar atención (Rulicki y Cherny, 2012). Estos autores plantean que casi todos los actos no verbales desempeñan una función reguladora, ya que pueden influir en la conducta de la audiencia, y su uso es, por consiguiente, deliberado y sobreentendido; se caracterizan por ser específicos de cada cultura y suele tratarse de señales muy sutiles, como el contacto ocular, la modificación de la postura corporal o los movimientos de cabeza.

Más allá de la sutileza de los actos no verbales, Poyatos (2004) destaca que generalmente nuestros interlocutores son más conscientes de nuestras conductas no verbales que nosotros mismos. El mismo autor distingue entre signos no verbales reguladores no segmentables y signos no verbales reguladores segmentables. En los primeros se ubican los 'marcadiscursos', que sirven para enfatizar o para mostrar la actitud frente a lo referido, 
mientras que en los segmentables se ubican, por ejemplo, un gesto manual o un elemento paralingüístico que permite llenar vacíos en el discurso. Este estudio se centra en los reguladores que presentan una función claramente estructuradora en los inicios de secuencia, ya sean segmentables o no.

\section{Metodología}

\subsection{Corpus}

En esta investigación se trabaja con base en 54 narraciones conversacionales de experiencia personal producidas, en parejas, por hablantes santiaguinos (Prieto, 19951996) del grupo de edad que va entre los 35 y los 54 años. El corpus de narraciones es parte del Proyecto FONDECYT No 11150007, titulado, "Entre la sociolingüística variacionista y la sociolingüística interaccional: un análisis génerolectal y sociolectal de la co-construcción de narraciones de experiencia personal". Dichas narraciones son coconstruidas, grabadas audiovisualmente, y obtenidas a través del diseño narrativo para el estudio autobiográfico y de tópico ${ }^{4}$.

\subsection{Población y muestra}

Como se precisó previamente, las narraciones fueron generadas por sujetos que conforman lo que en buena parte de los estudios sociolingüísticos del ámbito hispánico y, en concreto, los que siguen las directrices teórico metodológicas del Proyecto para el Estudio Sociolingüístico del Español de España y América (PRESEEA), se conoce como el segundo grupo etario, esto es, 35 a 54 años de edad. Esta decisión se sustenta en los hallazgos de Guerrero (2014), que apuntan a que este es el grupo de edad más sensible a la variación en la construcción de narraciones de experiencia personal generadas individualmente. La sociolingüística sugiere que el segundo grupo de edad (35 a 54 años) está confromado por los sujetos que tienen un desarrollo laboral pleno, puesto que se basa en las etapas vitales por las que pasa el hablante, las que suelen estar vinculadas con la cultura de cada comunidad lingüística (Blas Arroyo, 2005).

Respecto del tamaño muestral, se siguen las sugerencias de Moreno Fernández (1990) y Hernández Campoy y Almeida (2005). Se trabaja con una muestra por cuotas uniforme (López Morales, 1994), como se observa en la tabla 1.

Tabla 1. Tabla de distribución de los informantes de la muestra

\begin{tabular}{lllll}
\hline $\begin{array}{l}\text { Grupo } \\
\text { socioeconómico }\end{array}$ & $\begin{array}{l}\text { Hombre-Hombre } \\
(\mathrm{H}-\mathrm{H})\end{array}$ & $\begin{array}{l}\text { Hombre-Mujer } \\
(\mathrm{H}-\mathrm{M})\end{array}$ & $\begin{array}{l}\text { Mujer-Mujer } \\
\mathrm{M})\end{array}$ & $\begin{array}{l}\text { (M- } \\
\text { Bajo (B) }\end{array}$ \\
Medio (M) & 6 & 6 & 6 & 18 \\
Alto (A) & 6 & 6 & 6 & 18 \\
Totales & 18 & 6 & 6 & 18 \\
\hline
\end{tabular}

\footnotetext{
${ }^{4}$ Mertens (2005) divide en tres los estudios narrativos: a) de tópico, b) biográfico y c) autobiográficos. El diseño narrativo permite recopilar datos sobre historias de vida y experiencias personales para su descripción y análisis (Riessman 2008). Normas (ISSN: 2174-7245) | 


\subsection{Procedimiento de estratificación empleado}

Siguiendo el sistema de estratificación del Proyecto PRESEEA, la muestra se estratificó de acuerdo con sus respectivos niveles de instrucción, es decir, según los estudios básico (bajo), secundario (medio) y superior (alto). Sin embargo, con el propósito de garantizar la homogeneidad en la composición de los distintos grupos de la muestra y, por lo tanto, la representatividad de los mismos y su congruencia de estatus ${ }^{5}$ (Lenski, 1954), se complementó dicho sistema de estratificación con el procedimiento utilizado en el proyecto de Estudio Sociolingüístico del Español de Chile (ESECH) ${ }^{6}$. Dicho sistema de estratificación considera una escala de estatus socioeconómico que contempla las variables nivel educacional, categoría ocupacional y comuna de residencia (San Martín y Guerrero, 2015).

\subsection{Procedimiento analítico}

De acuerdo con los objetivos propuestos, el análisis se realizó de manera inductiva, siguiendo cuatro etapas: 1) observación de los reguladores no verbales de cierre de secuencia estructural de las narrativas, 2) determinación del catálogo de reguladores de cierre, 3) definición de cuáles son los reguladores más recurrentes y en qué parte de la cadena narrativa son más usados, 4) relación entre el empleo de reguladores no verbales de cierre con los factores sociodemográficos de los hablantes y 5) análisis contrastivo de los reguladores de inicio de secuencia estructural narrativa (Guerrero, 2017b) con los de cierre de secuencia.

Se tomó como unidad lingüística de análisis la cláusula final de las secuencias estructurales de cada narración y todos los reguladores no verbales involucrados en dicha cláusula. Esto implica que en un mismo momento del discurso puede haber más de un regulador, por ejemplo, la disminución en la velocidad del habla acompañada de una mirada hacia arriba y un arrugamiento del ceño. Se estudian las partes estructurales de inicio (resumen, si lo hay, y orientación), medio (complicación de la narración) y desenlace (resolución-coda) 7 . La evaluación, dado que es transversal en las narraciones y la primera vez que aparece suele coincidir con el inicio de la secuencia complicante, no se analiza; se sugiere que requiere un estudio independiente, puesto que, primero, no constituye un recurso estructural y, luego, no presenta signos reguladores, sino reguladores resaltadores de información relevante.

\section{PRESENTACIÓN, ANÁLISIS Y DISCUSIÓN DE LOS DATOS}

Esta sección del trabajo se subdivide en tres grandes partes. La primera incluye la clasificación y la caracterización de los reguladores no verbales de cierre de las secuencias estructurales de las narrativas que conforman la muestra, siguiente la propuesta teóricometodológica de Cestero (2016a). La segunda se hace cargo de presentar la distribución de los reguladores y de relacionarlos con las variables externas que se incorporan en esta

\footnotetext{
${ }^{5}$ Según Lenski (1954), se considera que un individuo es congruente con su estatus cuando las puntuaciones obtenidas en las diferentes dimensiones usadas para medir el estatus, son más o menos iguales, independiente de que sus rangos sean altos, bajos o estén en una extensión media. Cuando las puntuaciones del individuo son muy diferentes, se habla de incongruencia de estatus.

${ }^{6}$ En ESECH solo se habla de grupo Medio alto para los sujetos con educación universitaria o superior.

${ }^{7}$ En Guerrero (2017a) hemos estudiado la presencia y ausencia de las partes estructurales de las narrativas. Este análisis es la base para el que se propone en la investigación que aquí se presenta. En Moreno Fernández (2009) y en Guerrero y Muller (2016) se plantea que por su posición al final del relato, en muchas ocasiones resolución y coda tienden a fusionarse; sin embargo, también puede ocurrir que se presente solo resolución, solo coda, ninguna de las dos o ambas claramente delimitadas. Por esto, se ha decidido estudiar los reguladores no verbales de inicio de secuencia de cierre, sin segmentar entre resolución y coda. 
investigación y la última se encarga de presentar el análisis contrastivo del empleo de reguladores no verbales de inicio (Guerrero 2017b) y de cierre.

\subsection{Clasificación y caracterización de los reguladores no verbales de cierre de secuencia estructural narrativa}

El análisis inductivo de las 54 narraciones que analizamos sugiere los siguientes reguladores no verbales de cierre de secuencia estructural.

Tabla 2. Reguladores no verbales de cierre de secuencia estructural narrativa

\begin{tabular}{|c|c|c|c|}
\hline \multirow{7}{*}{ Paralenguaje } & \multirow{3}{*}{ Fónico } & \multirow{2}{*}{\multicolumn{2}{|c|}{$\begin{array}{l}\text { Tono (subida o bajada para llamar la atención del co-narrador y del } \\
\text { interlocutor) } \\
\text { Velocidad (+ o - velocidad para llamar la atención del co-narrador y del } \\
\text { interlocutor) }\end{array}$}} \\
\hline & & & \\
\hline & & \multicolumn{2}{|c|}{ Alargamiento (para llamar la atención o regular el discurso) } \\
\hline & \multirow{3}{*}{ Reacción psicológica } & \multicolumn{2}{|c|}{ Risa (muestra emoción frente a lo narrado) } \\
\hline & & \multicolumn{2}{|c|}{ Entre risas (muestra emoción frente a lo narrado) } \\
\hline & & \multicolumn{2}{|l|}{ Suspiro } \\
\hline & Pausas y silencios & \multicolumn{2}{|c|}{ Separadores (iniciales, que regulan) } \\
\hline \multirow{14}{*}{$\begin{array}{l}\text { Quinésica } 1 . \\
\text { Expresión facial }\end{array}$} & Sonrisa & \multicolumn{2}{|c|}{$\begin{array}{l}\text { Sonrisa leve (muestra emoción frente a lo narrado y simpatía con el co- } \\
\text { narrador) }\end{array}$} \\
\hline & \multirow{6}{*}{ Mirada } & \multicolumn{2}{|c|}{ Directa al co-narrador } \\
\hline & & \multicolumn{2}{|l|}{ Directa al interlocutor } \\
\hline & & \multicolumn{2}{|l|}{ Hacia abajo } \\
\hline & & Hacia arriba & \\
\hline & & En constante movimier & a de movimientos horizontales rápidos) \\
\hline & & $\begin{array}{l}\text { Hacia el horizonte (la } \\
\text { narrador con la historia }\end{array}$ & e desvía, mostrando involucramiento del \\
\hline & \multirow{7}{*}{ Marcadores } & \multicolumn{2}{|c|}{$\begin{array}{l}\text { Parpadeo lento (los ojos permanecen cerrados por más tiempo, } \\
\text { diferenciándose del parpadeo habitual) }\end{array}$} \\
\hline & & \multicolumn{2}{|c|}{$\begin{array}{l}\text { Parpadeo rápido (los ojos se cierran y abren de manera reiterada, más } \\
\text { rápido de lo habitual) }\end{array}$} \\
\hline & & \multicolumn{2}{|l|}{ Arrugamiento ceño } \\
\hline & & \multicolumn{2}{|l|}{ Arrugamiento nariz } \\
\hline & & \multicolumn{2}{|l|}{ Elevación de la ceja } \\
\hline & & \multirow{2}{*}{\multicolumn{2}{|c|}{$\begin{array}{l}\text { Gran abertura ojos (muestra emoción frente a lo narrador y llama la atención } \\
\text { del co-narrador y del interlocutor) } \\
\text { Movimiento o arrugamiento comisura boca (para tomar el turno y llamar la } \\
\text { atención del co-narrador y del interlocutor) }\end{array}$}} \\
\hline & & & \\
\hline \multirow{14}{*}{$\begin{array}{l}\text { Quinésica } 2 . \\
\text { Expresión corporal }\end{array}$} & \multirow{10}{*}{ Gestos } & \multirow{3}{*}{ Gestos con la cabeza } & Cabezadas \\
\hline & & & Giro cabeza-cuello horizontal \\
\hline & & & Giro cabeza-cuello horizontal constante \\
\hline & & \multirow{5}{*}{ Gestos manuales } & $\begin{array}{l}\text { Manotada (con una o con ambas } \\
\text { manos, vertical u horizontal, manos } \\
\text { semiabiertas. También pueden ser } \\
\text { constantes) }\end{array}$ \\
\hline & & & $\begin{array}{l}\text { Giro (con una o con ambas manos, } \\
\text { circular o semicircular) }\end{array}$ \\
\hline & & & $\begin{array}{l}\text { Giro constante (con una o con ambas } \\
\text { manos, circular o semicircular, de } \\
\text { manera permanente) }\end{array}$ \\
\hline & & & Levantamiento \\
\hline & & & Frotamiento-tocarse las manos \\
\hline & & \multirow{2}{*}{ Gestos corporales } & Balanceo cuerpo \\
\hline & & & Levantamiento de hombros \\
\hline & & \multicolumn{2}{|c|}{ Cruzar brazos quedando en postura fiia } \\
\hline & Pocturne & Entrelazar las manos qu & en postura fija \\
\hline & Posturas & Cruzar piernas quedan & tura fija \\
\hline & & Inclinación lateral cabe & quedando en postura fija \\
\hline & & Reorganización ocasiol & \\
\hline Proxémica & Comportamiento & Inclinación hacia delant & \\
\hline & & Inclinación hacia atrás & \\
\hline
\end{tabular}


Dado que una buena parte de los reguladores no verbales presentados se diferencia de aquellos incluidos en la investigación empírica de Cestero (2016a), así como de la de Cestero (2017), se puede señalar que la clasificación expuesta es representativa de los reguladores de cierre de secuencia estructural de narraciones de experiencia personal conversacionales. La mayor parte de ellos, sin embargo, coincide con los reguladores no verbales de inicio registrados por Guerrero (2017b), lo que podría implicar que se trata de una lista propia de marcas de regulación no verbal generadas en el marco de la narración cotidiana. Podemos señalar, entonces, que este trabajo constituye en parte lo que Poyatos (2003) denomina un "estudio realista del lenguaje".

Se encontraron, además, algunos gestos adaptadores, que funcionan específicamente como autorreguladores. Se incluyen en esta descripción porque regulan el cierre de secuencia estructural narrativa, aunque no son reguladores en el sentido estricto.

Tabla 3. Autorreguladores no verbales de cierre de secuencia estructural narrativa

\begin{tabular}{|c|c|c|}
\hline \multirow{5}{*}{ Adaptadores } & \multirow{5}{*}{ Autorreguladores } & Tocarse el pelo (autorregula mostrando inseguridad) \\
\hline & & $\begin{array}{l}\text { Tocarse la parte inferior de la cara (autorregula } \\
\text { mostrando inseguridad) }\end{array}$ \\
\hline & & Jugar con su ropa (autorregula mostrando inseguridad) \\
\hline & & $\begin{array}{l}\text { Jugar con sus dedos (autorregula mostrando } \\
\text { inseguridad) }\end{array}$ \\
\hline & & Jugar con objeto (autorregula mostrando inseguridad) \\
\hline
\end{tabular}

Los autorreguladores presentados en la tabla precedente presentan frecuencias de uso muy bajas, apenas 16 casos en total. El autorregulador más frecuente es jugar con algún objeto, como un papel o un lápiz, que se da en 7 ocasiones. Jugar con la ropa se registra en 4 oportunidades, tocarse el pelo y tocarse la parte inferior de la cara aparecen 2 veces cada uno y jugar con los dedos se aprecia una sola vez. Este último autorregulador se diferencia del gesto corporal tocarse-frotarse las manos en que, en el caso del autorregulador, el narrador se toma uno o dos dedos para jugar con ellos, moviéndolos de un lado a otro. Se trata de marcas muy específicas que, en las narraciones de la muestra, se pueden relacionar con la inseguridad de los hablantes, lo que se asocia, a la vez, son su nerviosismo y con su necesidad de estar moviéndose de manera constante. De hecho, estos autorreguladores aparecen, sobre todo, en la orientación y en la resolución-coda de la historia, es decir, o cuando está comenzando o cuando está finalizando.

\subsection{Distribución de los reguladores no verbales presentes al cierre del turno de la secuencia estructural narrativa}

Los hallazgos de Guerrero (2017a) sobre las partes estructurales de la narración señalan que la orientación se presenta en la totalidad de las narraciones de la muestra. La complicación del relato se incluye de manera explícita en más del 90\% de las narraciones, mientras que la resolución y la coda suelen presentarse entre el $70 \%$ y el $80 \%$ de las narraciones. Sin embargo, el resumen apenas supera el $40 \%$ de aparición en los grupos bajo y medio de la escala social, y se usa en un poco más del $70 \%$ en el grupo alto. En este sentido, el análisis de la distribución de los reguladores no verbales de cierre de secuencia estructural narrativa concuerda casi en su totalidad con los de Guerrero (2017a), ya que, en total, se registraron 573 casos de reguladores no verbales, distribuidos en 176/573 (30,7\%) en la complicación del relato, 171/573 (29,8\%) en la orientación, 152/573 $(26,5 \%)$ en el cierre del relato y $74 / 573$ (13\%) en el resumen. Se trata, en concreto, de 38 
tipos de reguladores de cierre distribuidos entre paralenguaje, expresión facial, expresión corporal y proxémica. Para informar de una manera más precisa sobre la distribución de los reguladores no verbales presentes al cierre de secuencia estructural narrativa, presentamos a continuación el análisis detallado. Cabe señalar que en la discusión de resultados solo se aludirá a aquellos casos llamativos, dado que los datos específicos se incluyen en la Tabla 4.

El empleo de reguladores no verbales de cierre en el resumen de las narraciones es escaso. Mayoritariamente se concentran en la mirada directa al interlocutor tras la cámara (11/74), como queriendo llamar su atención frente a lo que se está contando, y en el co-narrador (8/74), implicándolo en la narración. Al ser la parte inicial del relato, no es de extrañar que las miradas que buscan empatía e involucramiento con la audiencia sean recurrentes. También en el resumen la velocidad de habla es muy importante; se observa en 9/74 casos, en los que siempre se acelera la velocidad. Esto queda complementado con el cambio de tono (6/74) y con el cambio de la mirada hacia abajo (6/74). Con esto, el narrador da por finalizada la macroproposición de su historia. Sobre el empleo de reguladores no verbales en el cierre del resumen, también es pertinente precisar que de los 38 tipos registrados, solo se usan 23, por lo tanto, además de ser poco frecuentes son también poco diversos.

En lo que respecta a la orientación, los reguladores no verbales de cierre aumentan su cantidad y son muy diversos; hay apenas 6 tipos que no se emplean, lo que podría indicar que el narrador ya se siente cómodo y su discurso es, consecuentemente, más espontáneo. Nuevamente, las miradas juegan un rol trascendental, pero esta vez es la mirada directa al co-narrador la más relevante, pues se registra en 23/171 ocasiones. Por su parte, la mirada al interlocutor se observa en 16/171 oportunidades. A su vez, estas marcas del fin del relato de la orientación se caracterizan por ir acompañadas de un aumento de la velocidad del habla (17/171), de alargamientos (15/171), de manotadas (13/171), de sonrisas leves (11/171) y de pausas (8/171). En este sentido, puede argüirse que el proceso de pre-construcción narrativa descrito por Labov (2006) se está llevando a cabo con éxito y que los narradores, en la medida en que eligen las acciones que van a contar, se relajan y usan marcas que reflejan empatía con el interlocutor tras la cámara y con su co-narrador, como las sonrisas leves y las manotadas, que se utilizan porque se empieza a acercar el relato del clímax de la historia.

En relación con el uso de reguladores no verbales empleados en la complicación o clímax de la historia, cabe destacar que se utilizan 37/ 38 tipos de reguladores observados en el análisis, es decir, se produce un aumento, sobre todo, en la diversidad de marcas que van regulando los sucesos, aunque la frecuencia de empleo apenas supera a la de los reguladores observados en la orientación (176 versus 171). Se pone el énfasis, entonces, en los tipos de reguladores que caracterizan el discurso narrativo conversacional. En concreto, la velocidad de habla se enlentece o se acelera según lo que el narrador desee destacar (16/176). Las manotadas se vuelven más recurrentes todavía (14/176) y las miradas siguen siendo importantes (15/176 directa al co-narrador y 13/176 directa al interlocutor tras la cámara) y se suman la mirada en constante movimiento (10/176) y el discurso emitido entre risas (10/176) como reguladores clave en la historia, pues indicarían que el narrador está involucrado con los hechos e intenta captar la atención de su audiencia. Además, el cierre de la complicación de la historia se ve matizado con alargamientos y cambios de tono (8/176, de manera respectiva), que, responden, seguramente, a la necesidad de marcar partes cardinales del relato, generando cierta expectativa. Otros reguladores frecuentes son la risa, la sonrisa leve, la mirada hacia abajo, 
hacia el horizonte y el entrelazamiento de manos para quedar en postura fija al finalizar el climax de la narración (6 veces cada uno).

Por último, los reguladores no verbales presentes en el cierre de la parte final de la narrativa son la mirada directa al co-narrador (18/152) que puede reflejar, en medio de la sintonía narrativa con su compañero, que se da por finalizada la historia. También se produce un cambio en la velocidad del habla, que tiende a acelerarse (15/152) y las manotadas comienzan a reducirse respecto de la complicación de la historia (12/152). A su vez, se equiparan en su uso la mirada directa al interlocutor tras la cámara y la mirada hacia abajo (10/152), lo que también viene a marcar el cierre narrativo. A diferencia de la orientación y de la complicación, el parpadeo se vuelve más lento y hay más cabezadas que tienden a asentir, y menos manotadas, que señalan que la historia llegó a su fin.

A partir de la síntesis de hallazgos esbozada, se puede precisar que existen algunos reguladores secuenciales de cierre en las narrativas interaccionales bien definidos. Se destaca especialmente el papel que tienen las miradas en todas las secciones de la narración y es relevante, además, que existan algunos reguladores no verbales muy frecuentes y diversos en el clímax del relato, a la vez que es interesante que, en cuanto a frecuencia, en algunos casos en que son coincidentes, tanto en el resumen como en la parte final de la historia, tienden a tener comportamientos similares, inclinándose a la disminución. La Tabla 4 incluye el detalle de lo recientemente expuesto. 
Los reguladores no verbales de inicio y de cierre...| S. Guerrero

Tabla 4. Síntesis de empleo de reguladores no verbales de cierre de secuencia estructural narrativa

\begin{tabular}{|c|c|c|c|c|c|c|}
\hline \multirow{2}{*}{\multicolumn{2}{|c|}{ Reguladores no verbales }} & \multicolumn{4}{|c|}{ Secuencia estructural narrativa } & \multirow{3}{*}{$\begin{array}{l}\text { Total } \\
\text { empleo } \\
\text { reguladores } \\
\text { de cierre } \\
30\end{array}$} \\
\hline & & \multirow{2}{*}{$\begin{array}{l}\text { Resumen } \\
6\end{array}$} & \multirow{2}{*}{$\begin{array}{l}\text { Orientación } \\
7\end{array}$} & \multirow{2}{*}{$\begin{array}{l}\text { Complicación } \\
8\end{array}$} & \multirow{2}{*}{$\begin{array}{l}\text { Cierre } \\
9\end{array}$} & \\
\hline \multirow{7}{*}{ 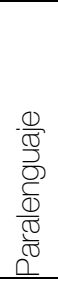 } & Tono (subida o baiada) & & & & & \\
\hline & Velocidad (+ o- velocidad) & 9 & 17 & 16 & 15 & 57 \\
\hline & Alargamiento & 2 & 15 & 8 & 3 & 28 \\
\hline & Risa & 0 & 0 & 6 & 3 & 9 \\
\hline & Entre risas & 0 & 3 & 10 & 7 & 20 \\
\hline & Suspiro & 0 & 0 & 1 & 0 & 1 \\
\hline & Separadores (iniciales) & 1 & 8 & 2 & 3 & 14 \\
\hline \multirow{14}{*}{ 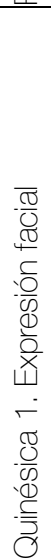 } & Sonrisa leve & 5 & 11 & 6 & 6 & 28 \\
\hline & Mirada directa al interlocutor & 11 & 16 & 13 & 10 & 50 \\
\hline & Mirada directa al co-narrador & 8 & 23 & 15 & 18 & 64 \\
\hline & Mirada hacia abajo & 6 & 5 & 6 & 10 & 27 \\
\hline & Mirada hacia arriba & 0 & 1 & 4 & 1 & 6 \\
\hline & Mirada hacia el horizonte & 4 & 7 & 6 & 5 & 22 \\
\hline & Mirada constante & 0 & 6 & 10 & 7 & 23 \\
\hline & Parpadeo lento & 0 & 1 & 1 & 3 & 5 \\
\hline & Parpadeo rápido & 0 & 1 & 1 & 1 & 3 \\
\hline & Arrugamiento ceño & 1 & 3 & 1 & 3 & 8 \\
\hline & Elevación de la ceja & 0 & 1 & 1 & 0 & 2 \\
\hline & Arrugamiento nariz & 0 & 2 & 0 & 3 & 5 \\
\hline & Movimiento comisura boca & 0 & 0 & 0 & 1 & 1 \\
\hline & Gran abertura ojos & 0 & 0 & 2 & 1 & 3 \\
\hline \multirow{14}{*}{ 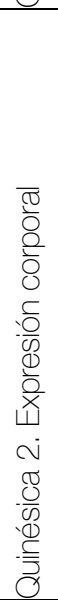 } & Cabezadas & 0 & 1 & 3 & 8 & 12 \\
\hline & Giro cabeza-cuello & 0 & 2 & 2 & 3 & 7 \\
\hline & Giro cabeza-cuello constante & 0 & 1 & 0 & 2 & 3 \\
\hline & Manotada & 3 & 13 & 14 & 12 & 42 \\
\hline & Giro manual & 1 & 7 & 5 & 7 & 20 \\
\hline & Giro manual constante & 1 & 1 & 4 & 0 & 6 \\
\hline & Levantamiento manual & 1 & 0 & 1 & 0 & 2 \\
\hline & Frotamiento manual & 1 & 1 & 4 & 2 & 8 \\
\hline & Balanceo cuerpo & 1 & 2 & 2 & 2 & 7 \\
\hline & Levantamiento de hombros & 2 & 2 & 3 & 0 & 7 \\
\hline & Cruzar brazos & 1 & 1 & 1 & 1 & 4 \\
\hline & Entrelazar manos & 3 & 5 & 6 & 6 & 20 \\
\hline & Cruzar piernas & 0 & 1 & 1 & 0 & 2 \\
\hline & $\begin{array}{l}\text { Inclinación lateral cabeza- } \\
\text { cuello }\end{array}$ & 2 & 3 & 1 & 3 & 9 \\
\hline \multirow{3}{*}{ 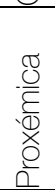 } & Reorganización & 4 & 2 & 4 & 0 & 10 \\
\hline & Inclinación hacia adelante & 1 & 2 & 4 & 0 & 7 \\
\hline & Inclinación hacia atrás & 0 & 3 & 0 & 0 & 3 \\
\hline
\end{tabular}

\subsubsection{Relación del empleo de los reguladores no verbales de cierre con los factores sexo y grupo socioeconómico}

El propósito de esta sección del estudio es intentar describir las identidades de los hablantes, tomando como base los postulados de Georgakopoulou (2006), quien señala que hay que poner el énfasis en el surgimiento de identidades en el discurso, particularmente en sitios interactivos, donde pueden presentar una multiplicidad de significados. Se sintetizan, entonces, los hallazgos relativos al análisis descriptivo del comportamiento de los reguladores no verbales de cierre de secuencia estructural narrativa 
en relación con las variables sexo y grupo socioeconómico de los informantes. Respecto del sexo, se estudian tres niveles: hombre-hombre $(\mathrm{H}-\mathrm{H})$, hombre-mujer $(\mathrm{H}-\mathrm{M})$ y mujermujer (M-M), y en relación el con grupo socioeconómico se analizan los niveles bajo, medio y alto.

Tabla 5. Relación del empleo de reguladores no verbales de cierre con los factores sexo y grupo socioeconómico

\begin{tabular}{|l|l|l|l|l|}
\hline $\begin{array}{l}\text { Secuencia } \\
\text { estructural narrativa }\end{array}$ & Sexo & $\begin{array}{l}\text { Frecuencia empleo } \\
\text { reguladores de cierre }\end{array}$ & GSE & $\begin{array}{l}\text { Frecuencia empleo } \\
\text { reguladores de cierre }\end{array}$ \\
\hline \multirow{3}{*}{ Resumen } & $\mathrm{H}-\mathrm{H}$ & $26 / 74$ & Bajo & $31 / 74$ \\
\cline { 2 - 6 } & $\mathrm{H}-\mathrm{M}$ & $21 / 74$ & Medio & $25 / 74$ \\
\cline { 2 - 6 } & $\mathrm{M}-\mathrm{M}$ & $27 / 74$ & Alto & $18 / 74$ \\
\hline \multirow{3}{*}{ Orientación } & $\mathrm{H}-\mathrm{H}$ & $58 / 171$ & Bajo & $58 / 171$ \\
\cline { 2 - 6 } & $\mathrm{H}-\mathrm{M}$ & $56 / 171$ & Medio & $59 / 171$ \\
\cline { 2 - 6 } & $\mathrm{M}-\mathrm{M}$ & $61 / 171$ & Alto & $54 / 171$ \\
\hline \multirow{3}{*}{ Complicación } & $\mathrm{H}-\mathrm{H}$ & $56 / 176$ & Bajo & $63 / 176$ \\
\cline { 2 - 6 } & $\mathrm{H}-\mathrm{M}$ & $59 / 176$ & Medio & $55 / 176$ \\
\cline { 2 - 6 } & $\mathrm{M}-\mathrm{M}$ & $61 / 176$ & Alto & $58 / 176$ \\
\hline \multirow{3}{*}{ Cierre } & $\mathrm{H}-\mathrm{H}$ & $54 / 152$ & Bajo & $50 / 152$ \\
\cline { 2 - 5 } & $\mathrm{H}-\mathrm{M}$ & $49 / 152$ & Medio & $58 / 152$ \\
\cline { 2 - 5 } & $\mathrm{M}-\mathrm{M}$ & $49 / 152$ & Alto & $44 / 152$ \\
\hline
\end{tabular}

Los datos sintetizados reflejan que, en el caso del resumen, las parejas conformadas por hablantes del mismo sexo se comportan de manera similar, en tanto que la pareja mixta disminuye la frecuencia de empleo de reguladores no verbales de cierre. En cuanto al grupo socioeconómico, puede decirse que son los hablantes del grupo bajo lo que más emplean reguladores no verbales de cierre y los del alto los que menos lo hacen.

En relación con la orientación del relato, son las parejas de mujeres las que más usan reguladores no verbales de cierre, mientras que las de hombres y las mixtas no difieren mucho en su comportamiento. Sobre el grupo socioeconómico, los tres grupos se comportan de manera similar, con una leve disminución en la frecuencia de empleo en el grupo alto.

Luego, en la complicación de la narración, puede observarse que son nuevamente las parejas de mujeres las que más usan reguladores de cierre y, específicamente, las del grupo bajo de la escala social. El resto de los hablantes presentan frecuencias muy similares.

Por último, en la secuencia de cierre (resolución-coda) del relato, son las parejas de hombres las que se destacan por el uso de reguladores no verbales de cierre narrativo y, en específico, aquellos del grupo medio. Respecto del grupo socioeconómico, se observan 14 puntos de diferencia entre el grupo que menos regulares emplea -el alto- y el que más los usa -el medio-.

En definitiva, puede decirse que es en la parte final de la cadena narrativa (resolución-coda) donde se observan las principales diferencias de empleo de reguladores no verbales de cierre y donde los hablantes divergen en su comportamiento respecto de las otras partes de la secuencia de la narración. A la vez, es pertinente precisar que parece ser que son las mujeres de los grupos medio y bajo de la escala social las que más utilizan reguladores de 
cierre, lo que podría marcar su simpatía o empatía e involucramiento con la audiencia. En relación con estos hallazgos, Tannen (2011: 136) postula "Why are women more attuned to metamessages? Because they are more focused on involvement, that is, on relationships among people are established and maintained". Esto viene a reforzar la idea de que el estilo conversacional de las mujeres es más colaborador e involucrado que el de los hombres (Tannen, 2017).

Si bien los resultados de frecuencia y las observaciones previas obedecen a un enfoque descriptivo, se trata de hallazgos que nos parecen suficientes para dar cuenta de los patrones de empleo de reguladores no verbales de cierre de secuencia estructural narrativa.

\subsection{Comparación del empleo de los reguladores no verbales de inicio y de cierre del turno de la secuencia estructural narrativa}

En esta tercera parte del análisis, el primer dato necesario de precisar es que se registraron 38 tipos de reguladores no verbales de cierre versus 31 de inicio. Estos últimos han sido descritos en Guerrero (2017b). Se trata, concretamente, de 573 marcas de cierre y de 492 de inicio, por lo tanto, puede decirse que los reguladores no verbales de cierre son más variados y más frecuentes que los de inicio de secuencia estructural narrativa. A nivel de paralenguaje, es destacable la presencia de los suspiros, en tanto reacción psicológica de cierre. Este tipo de regulador no se encontró entre las marcas de inicio. En cuanto a la expresión facial, se agregan el parpadeo rápido, el arrugamiento del ceño y el arrugamiento de la nariz como reguladores propios de los cierres narrativos. Asimismo, en la expresión corporal se destacan las cabezadas, el giro manual constante y el levantamiento de hombros, a la vez que se agregan la postura de cruzar las piernas para quedar en postura fija.

De los reguladores de inicio de secuencia narrativa que no se registran al cierre de esta, es destacable el comportamiento proxémico de tocar al interlocutor.

En lo que respecta a los autorreguladores, se agregan la posibilidad de jugar con la ropa, jugar con los dedos y jugar con un objeto, como marcas propias de los cierres narrativos. Este hallazgo se suma a tocarse el pelo y tocarse la parte inferior de la barbilla, que ya habían sido incluidos en el catálogo de reguladores no verbales de inicio, que, a su vez, se presentan entre los reguladores de cierre.

Al realizar el contraste da datos con los de Guerrero (2017b), se observa que excepto en el resumen, los reguladores de cierre de secuencia narrativa son siempre más frecuentes que los de inicio. A este respecto, solo se han considerado los casos en que había coincidencia, en el tipo de regulador no verbal y solo se incluyen observaciones respecto del total de casos. Así, en relación con el paralenguaje, solo los alargamientos son más frecuentes en tanto reguladores de inicio, lo que no es extraño en la medida en que los informantes están organizando las historias en sus mentes y van pensando y seleccionando aquellos episodios que relatarán; los alargamientos reflejan este proceso mental. En cuanto a la expresión facial, entre otros casos, es interesante que las miradas directas al interlocutor, hacia arriba y hacia el horizonte también son más frecuentes al inicio de la secuencia estructural narrativa, probablemente, porque los narradores están buscando empatía con sus co-narradores; sobre todo las miradas mutuas generan esta integración y aumentan el grado de involucramiento. 
En el caso de la expresión corporal, tanto el giro de la cabeza-cuello y el giro manual son más recurrentes al inicio de las secuencias estructurales narrativas, pero las manotadas y el entrelazamiento de manos para quedar en postura fija son muy típicas de los cierres; estos últimos, en especial, indican que la cláusula narrativa ha llegado a su fin.

Por último, en cuanto a los reguladores no verbales correspondientes a la proxémica, siempre hay mayor frecuencia al inicio de secuencias narrativas. Esto implica que los hablantes se están reorganizado de manera permanente, y se inclinan hacia adelante 0 hacia atrás para comentar una parte estructural del relato. Hacia el final de las historias, están en posturas más fijas y, en consecuencia, los narradores se acomodan menos ${ }^{8}$.

Pese a que no era el objetivo de este trabajo, para complementar el análisis inductivo que posibilitó este contraste de datos, se realizaron algunas pruebas estadísticas. Los datos fueron procesados con el software R (R Core team, 2015; Wickham, 2009; Zeileis, David y Hornik, 2007). Tomando en consideración que los rasgos lingüísticos escogidos fueron medidos en términos dicotómicos (presencia o ausencia), los análisis estadísticos se efectuaron recurriendo a estadística no paramétrica, esto es, un modelo loglineal para las tablas de contingencia de tres vías. Tomando como variable respuesta la presencia/ausencia de cada rasgo lingüístico, se efectuaron análisis tomando siempre como variable independiente el factor inicio/cierre y alternando como variables independientes: a) las diadas de sexo, b) el grupo socioeconómico y c) los rasgos de la estructura narrativa. Solo se reportarán los casos más relevantes.

En relación con el factor sexo, resultó significativa, entre otras, la comparación de presencia y ausencia de reguladores no verbales de inicio y de cierre denominado entre risas, más frecuente en los cierres secuenciales narrativos de hombre-hombre ( $p=0,001)$; las manotadas $(p=0,01)$, también más recurrentes en los cierres secuenciales de las narraciones de hombre-hombre; la reorganización ocasional, que en las secuencias de cierre se comporta de manera casi idéntica, pero en las cláusulas iniciales de secuencias de inicio aumenta su uso, sobre todo, en las parejas hombre-hombre, con un valor $p=0,01$; $y$, por último, el cambio de tono ( $p=0,008)$, más común en los cierres secuenciales de las narrativas de parejas hombre-mujer.

En cuanto al factor grupo socioeconómico, se destacan, especialmente, el caso del regulador no verbal entre risas ( $p=0,004)$, más frecuente entre los reguladores no verbales de cierre secuencial del grupo medio de la escala social; los giros cabeza-cuello, muy comunes en las cláusulas de inicio de secuencias narrativas $(p=0,01)$, en particular, en el grupo bajo; la mirada hacia arriba $(p=0,02)$, también más común en las cláusulas de inicio de los hablantes del grupo bajo; las manotadas $(p=0,005)$, que aparecen en cláusulas de cierre con mayor frecuencia que al inicio, y que suelen aparecer mucho en el grupo bajo y muy escasamente en los inicios narrativos de los hablantes del grupo alto de la escala social; el movimiento de la comisura de la boca $(p=0,03)$, que se registra muy poco, pero es más común en las cláusulas de inicio narrativo del grupo bajo; la reorganización ocasional $(p=0,03)$, que se da más generalmente entre los reguladores no verbales de inicio de secuencia estructural narrativa y en los de cierre disminuye su uso, sobre todo, en el grupo de narradores del nivel alto de la escala social; $y$, el cambio de tono ( $p=0,006)$, más

8 Para revisar las frecuencias de uso específicas de reguladores no verbales de inicio de secuencia narrativa, ver Guerrero (2017b). 
frecuente en los cierres que en los inicios, y casi inexistente en las cláusulas de inicio de secuencia narrativa entre los hablantes del grupo alto.

La asociación de la presencia o ausencia de reguladores no verbales de inicio y cierre con el rasgo de la estructura narrativa (resumen, orientación, complicación y resolución-coda), permite señalar que los alargamientos son estadísticamente significativos $(p=0,02)$, más frecuentes en las cláusulas de cierre de la orientación; la mirada directa al co-narrador $(p=0,03)$, más usada en cláusulas de inicio de la orientación; la mirada directa al interlocutor $(p=0,01)$, que suele utilizarse de manera similar tanto en cláusulas de inicio como de cierre de secuencias narrativas, pero disminuye su empleo en los inicios de la resolución-coda; los giros cabeza-cuello ( $p=0,02)$, más frecuentes en los inicios de la orientación narrativa; los giros cabeza-cuello constantes ( $p=0,02)$, que casi no se registran, pero se suelen usar en las cláusulas de inicio de la resolución-coda; el levantamiento de manos $(p=0,02)$, también utilizado en cláusulas de inicio de la resolución-coda; las manotadas ( $p=0,002)$, más comunes en las cláusulas de cierre tanto de la orientación como de la complicación; la reorganización ocasional ( $p=0,003)$, que se presenta más en las cláusulas de inicio de la orientación y disminuye su frecuencia en los cierres de la resolución-coda; y, los cambios de tono $(p=0,05)$, que difiere en su uso entre las cláusulas de inicio y de cierre narrativo de la resolución-coda, es decir, no se usa al inicio y aumenta su uso en los cierres.

En síntesis, son las narraciones generadas por hombre-hombre las que muestran datos más relevantes, sobre todo, si son del grupo social bajo. Además, es en la orientación y en la complicación de las historias donde se aprecian más casos de variación, seguramente, porque son también las partes estructurales que presentan frecuencias de uso más elevadas. Los casos más frecuentes de variación son el regulador entre risas, el cambio de tono y la reorganización ocasional.

\section{CONCLUSIONES}

Los principales hallazgos del estudio pueden sintetizarse como sigue:

Del mismo modo que se demostró en Guerrero (2017b) con los reguladores no verbales de inicio de secuencia estructural narrativa, se puede señalar que es posible proponer una categorización de reguladores no verbales de cierre, pues se identifican 38 reguladores y cinco autorreguladores concretos, que marcan el final de cada parte de la cadena estructural de la narración.

En lo que respecta a la distribución de estos reguladores en la cadena secuencial narrativa, puede decirse que existen algunos claramente definidos. Se destaca especialmente el papel que tienen las miradas en todas las secciones de la narración y es relevante, asimismo, que existan algunos reguladores no verbales muy recurrentes y diversos en el cierre de la parte climática del relato. Además, es interesante que, en cuanto a su frecuencia, en algunos casos en que se emplea el mismo tipo de regulador, tanto en el resumen como en la complicación-coda de la historia, suele haber comportamientos similares, tendiendo a la disminución de frecuencia de uso.

La relación con los factores sexo y grupo socioeconómico de los hablantes, permite concluir, en términos descriptivos y sobre el total de datos, que los reguladores no verbales de cierre de secuencia estructural de la narración suelen utilizarse más por las parejas de narradoras mujeres, excepto en la sección resolución-coda, donde destaca la frecuencia 
de empleo de las parejas hombre-hombre, y que son más comunes entre los hablantes de los grupos bajo y medio de la escala social.

Las pruebas estadísticas de comparación de empleo de reguladores específicos señalan que son los hablantes hombre-hombre los que presentan los datos más interesantes y, en especial, aquellos del grupo bajo de la escala social. Asimismo, el análisis de asociación de la presencia o ausencia de reguladores no verbales de inicio y cierre con el rasgo de la estructura narrativa permite concluir que los casos estadísticamente significativos son los que se vinculan con la orientación y con la resolución-coda de las narraciones conversacionales.

\section{REFERENCIAS BIBLIOGRÁFICAS}

Álvarez de Arcaya AJuRIA, Helena (2003): «La comunicación no verbal. Interrelaciones entre las expresiones faciales innatas y las aprendidas», Gazeta de Antropología, 19, 110.

BLAS ARROYo, José Luis (2005): Sociolingüística del español. Desarrollos y perspectivas en el estudio de la lengua española en contexto social, Madrid, Cátedra.

ANDREWS, Molly, Corinne SquiRE y María TAMBOUKOU (2013): Doing narrative research, LA, Sage Publications Ltd.

Cestero, Ana María (2004): «La comunicación no verbal y el aprendizaje de lenguas extranjeras", en Sánchez Lobato, Jesús e Isabel Santos Gargallo, dirs., Vademécum para la formación de profesores. Enseñar español como segunda lengua/lengua extranjera, Madrid, SGEL, 593616.

Cestero, Ana María (2009): «Marcas paralingüísticas y quinésicas de la ironía», en Ruiz Gurillo, Leonor y Xosé Padilla García, eds., Dime cómo ironizas y te diré quién eres. Una aproximación pragmática a la ironía, Frankfurt/Main, Peter Lang, 167-190.

Cestero, Ana María (2014): "Comunicación no verbal y comunicación eficaz", ELUA Estudios de Lingüística, 28, 125-150.

Cestero, Ana María (2016a): «La Comunicación no verbal: propuestas metodológicas para su estudio», Lingüística en la Red, 2-36 [en línea]: http://www.linred.es/. [Consulta: 20/11/2017].

Cestero, Ana María (2016b): «La atenuación en el habla de Madrid: patrones sociopragmáticos», RILCE, 33,1, 57-86.

Cestero, Ana María (2017): «La comunicación no verbal en discurso persuasivo empresarial», Pragmalingüística, 25, 124-145.

CHEEPEN, Christine (1988): The predictability of informal conversation, London, Pinter.

EKMAN, Paul (1973): "Cross cultural studies of facial expression», en Ekman, Paul, ed., Darwin and facial expression, New York, Academic Press, 169-222.

EKMAN, Paul y Wallece Friesen (1969): «The repertoire of non-verbal behaviour: categories, origins, usage and coding", Semiotica, 1, 4998.

GEORGAKOPOULOU, Alexandra (2006): «Small and large identities in narrative (inter)action», en De Fina, Anna, Deborah Schiffrin y Michael Bamberg, eds., Discourse and identity, Cambridge, Cambridge University Press, 83102, https://doi.org/10.1017/CBO9780511584459

GUERRERO, Silvana (2014): Variación discursiva en narraciones de experiencia personal en el español hablado en Santiago de Chile, Tesis doctoral, Chile, Pontificia Universidad Católica de Chile.

GUERRERO, Silvana (2017a): «Narración individual versus narración conversacional: un puente entre el variacionismo y la interacción", Onomázei, 38, 58-87.

GUERRERO, Silvana (2017b): «Los reguladores no verbales de inicio de secuencia estructural en narraciones conversacionales: un estudio sociopragmático», Pragmalingüística, 25, 267285.

GueRRERO, Silvana y Valeska MulleR (2016): «La 'coda' narrativa: una propuesta de clasificación y aplicación al estudio variacionista en el español chileno", Lenguas Modernas, 48, 2946.

GoodWIN, Charles (2007): «Participation, stance and affect in the organization of activities", Discourse \& Society, 18 (1), 53-73.

HeRnÁNDEZ-CAMPOY, Juan Manuel y Manuel AlmeIDA (2005): Metodología de la investigación sociolingüística, Málaga, Editorial Comares.

JUZWIK, Mary (2012): «Spoken Narrative», en Gee, James y Michael Handford, eds., The Routledge Handbook of Discourse Analysis, Routledge Handbooks in Applied Linguistics, London, Routledge, 326-341.

LABOV, William (1972): "The transformation of experience in narrative syntax», Language in the inner city. Studies in the Black English Vernacular, Filadelfia, University of Pennsylvania Press, 354-396.

LABOV, William (2006): "Narrative preconstruction», Narrative Inquiry, 1, 37-45. 
LABOV, William (2013): The language of life and death. The transformation of experience in oral narrative, Cambridge, University Press.

LABOV, William y Joshua WALETZKY (1967): «Narrative analysis», en Helm, June, ed., Essays on the verbal and visual arts, Seattle, University of Washington Press, 12-44.

LENSKI, Gerhard (1954): "Status crystalization: A non-vertical dimension of social status», American Sociological Review, 19, 405-413.

LÓPEZ MORALES, Humberto (1994): Métodos de investigación lingüística, Salamanca, Ediciones Colegio de España.

MERTENS, Donna (2005): Research and Evaluation in Education and Psychology: Integrating Diversity With Quantitative, Qualitative, and Mixed Methods, Thusand Oaks, Sage.

MoRENO FERNÁNDEZ, Francisco (1990): Metodología sociolingüística, Madrid, Gredos.

MoReno FeRnándeZ, Francisco (2009): Principios de sociolingüística y sociología del lenguaje, Barcelona, Ariel.

NoRRICK, Nick (2000): Conversational narrative, Saarland University, John Benjamins Publishing Company.

POYATOS, Fernando (2003): «La comunicación no verbal. Algunas de sus perspectivas de estudio e investigación», Revista de investigación lingüística, 6, 2, 67-84.

POYATOS, Fernando (2004): «Los comportamientos no verbales y su consideración en el aula», Puertas de lectura, 17, 116-127.

Prieto Vera, Luis (1995-1996): «Análisis sociolingüístico del dequeísmo en el habla de Santiago de Chile», Boletín de Filología, Tomo XXXV, 379-452.

R CoRE TEAm (2015): R: A Language and Environment for Statistical Computing, [en línea]: http://www.R-project.org/ [Consulta: 10/01/2017].
RIESSMAN, Catherine (2008): Narrative methods for the human sciences, CA, USA, Sage Publications, Inc.

RULICKI, Sergio y Martin CHERNY (2012): Comunicación no-verbal: cómo la inteligencia emocional se expresa a través de los gestos, Mishawaka, Ediciones Granica S.A

SAN MARTín, Abelardo y Silvana GueRRERO (2015): «Estudio sociolingüístico del español de Chile (ESECH): recogida y estratificación del corpus de Santiago", Boletín de Filología, 50 (1), 221 247.

SIDNELL, Jack (2010): Conversation Analysis: An introduction, UK, Willey-Blackwell.

SILVA-CORVALÁN, Carmen (2001): Sociolingüística y pragmática del español, Washington, Georgetown University Press.

TANNEN, Deborah (2011): That's not what I meant!, New York, HarperCollins Publishers.

TANNEN, Deborah (2017): You're the only one I can tell. Inside the language of women's friendships, New York, Ballantine Books.

THORNBORROW, Joanna y Jennifer COATES (2005): The sociolinguistics of narrative, Amsterdam/Philadelphia, John Benjamins Publishing Company.

Tusón, Amparo (1995): Análisis de la conversación, Barcelona, Ariel.

VAN DIJK, Teum (1997): «Historias y racismo», Mumby, Dennis, comp., Narrativa y control social. Perspectivas críticas, Buenos Aires, Amarrortu Editores, 163-190.

WiCKHAM, Hadley (2009): ggplot2: Elegant Graphics for Data Analysis, Dordrecht/New York, Springer.

ZEILEIS, Achim, Meyer DAVID y Hornik KURT (2007): «Residual-based Shadings for Visualizing (Conditional) Independence», Journal of Computational and Graphical Statistics, 16 (3), 507-525. 